Revealing the potential of squid chitosan-based structures for biomedical applications

This article has been downloaded from IOPscience. Please scroll down to see the full text article.

2013 Biomed. Mater. 8045002

(http://iopscience.iop.org/1748-605X/8/4/045002)

View the table of contents for this issue, or go to the journal homepage for more

Download details:

IP Address: 193.137.16.117

The article was downloaded on 30/05/2013 at 10:12

Please note that terms and conditions apply. 


\title{
Revealing the potential of squid chitosan-based structures for biomedical applications
}

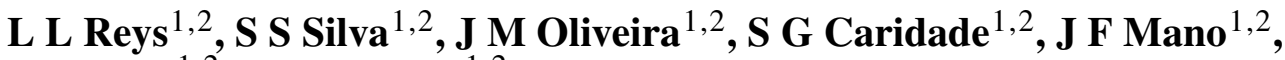 \\ T H Silva ${ }^{1,2}$ and R L Reis ${ }^{1,2}$ \\ 1 3Bs Research Group-Biomaterials, Biodegradables and Biomimetics, University of Minho, \\ Headquarters of the European Institute of Excellence on Tissue Engineering and Regenerative Medicine, \\ AvePark, 4806-909 Taipas, Guimarães, Portugal \\ ${ }^{2}$ ICVS/3Bs-PT Government Associate Laboratory, Braga/Guimarães, Portugal \\ E-mail: lara.reys@ dep.uminho.pt
}

Received 23 October 2012

Accepted for publication 16 April 2013

Published 28 May 2013

Online at stacks.iop.org/BMM/8/045002

\begin{abstract}
In recent years, much attention has been given to different marine organisms, namely as potential sources of valuable materials with a vast range of properties and characteristics. In this work, $\beta$-chitin was isolated from the endoskeleton of the giant squid Dosidicus gigas and further deacetylated to produce chitosan. Then, the squid chitosan was processed into membranes and scaffolds using solvent casting and freeze-drying, respectively, to assess their potential biomedical application. The developed membranes have shown to be stiffer and less hydrophobic than those obtained with commercial chitosan. On the other hand, the morphological characterization of the developed scaffolds, by SEM and micro-computed tomography, revealed that the matrices were formed with a lamellar structure. The findings also indicated that the treatment with ethanol prior to neutralization with sodium hydroxide caused the formation of larger pores and loss of some lamellar features. The in vitro cell culture study has shown that all chitosan scaffolds exhibited a non-cytotoxic effect over the mouse fibroblast-like cell line, L929 cells. Thus, chitosan produced from the endoskeletons of the giant squid Dosidicus gigas has proven to be a valuable alternative to existing commercial materials when considering its use as biomaterial.
\end{abstract}

\section{Introduction}

The efficient exploitation of natural resources is a critical issue: on the one hand, the chemical and biological diversity of nature is uncountable, but on the other, natural resources are limited and must be fully exploited. From this perspective, the valorization of natural polymers is becoming increasingly important for technological and industrial-related applications [1], particularly polymers that can be obtained from marine organisms [2]. At the same time, great efforts into the research of natural polymers are being made in the biomedical field, due to their intrinsic properties and availability in nature [1].

Since 1990, biomedical studies on chitin (CT) and chitosan (CHT) have intensified due to their excellent biological properties such as biodegradation in the human body and immunological, antibacterial and wound-healing activity [3]. In particular, reports on applications such as drug delivery systems, wound dressings, tissue engineering, ophthalmology systems and others can be found in the literature [4-8].

CT, the second most abundant natural polymer after cellulose, is an ideally (1-4)-linked polysaccharide composed of 2-acetoamide-2-deoxy- $\beta$-D-glucopyranosyl residues, while CHT is its $\mathrm{N}$-deacetylated derivative [9]. CT can be extracted from a wide range of natural sources such as crustaceans, fungi, insects, mollusks and some algae [10]. Depending on the source, it can exhibit one of two allotropic forms: $\alpha$ and $\beta$. For instance, $\alpha$-chitin can be found in crustacean shells, while 
$\beta$-chitin is present in squid pens [11]. So far, little attention has been given to the $\beta$-crystallographic form, mainly due to the difficulty in obtaining the product; because of this CHT has been commercially produced almost exclusively from $\alpha$-chitin $[10,11]$. Despite the above-mentioned properties that make them quite interesting biomedically, both CT and CHT exhibit limiting factors in their reactivity and processability; they are not water soluble, degrade thermally and it is difficult to convert $\mathrm{CT}$ into $\mathrm{CHT}$ by deacetylation $[11,12]$. $\beta$-chitin has weaker intermolecular bonds, higher reactivity and higher affinity for solvents when compared to $\alpha$-chitin. Besides this, CHT prepared from $\beta$-chitin also exhibits higher reactivity and significant bactericidal activity [10, 13]. Thus, CHT derived from $\beta$-chitin obtained from squid pens may overcome some of the aforementioned drawbacks. Moreover, the increase in the valorization of marine resources is resulting in the increase of the raw material for $\beta$-chitin production. In fact the waste from food processing industries, namely the endoskeletons of cephalopods, could be both economically and environmentally valuable asa resource for obtaining CT. The aim of the present work consists of the isolation and the physicochemical and biological characterization of $\beta$-chitin obtained from the squid pen of Dosidicus gigas (a giant squid). The CT was isolated from the mentioned squid specie using the method based on the work of Chaussard and Domard [14], who evaluated the various physicochemical parameters that can influence the production of CT from a sample of squid pens (loligo). Further, the extracted $\beta$-CT was converted to CHT through a deacetylation reaction. Then, squid chitosan (CHTS) was processed as membranes and scaffolds using the solvent casting and freeze-drying techniques, respectively. These matrices were characterized by their mechanical properties, swelling behaviour, structural stability and cytotoxicity.

\section{Materials and methods}

\subsection{Materials}

Squid pens from Dosidicus gigas, used as raw material for $\beta$-chitin isolation, were obtained from individuals captured on the coast of Chile and kindly provided by PESCANOVA. Commercial chitosan (CHTC, Sigma Aldrich, CAS 9012-76-4) with medium molecular weight was used after purification. Commercial chitin (CTC, Sigma Aldrich) was used as received. All other reagents, of analytical grade, were used as received.

\subsection{Production of squid chitosan}

2.2.1. Isolation of chitin from squid pens. Isolation of $\beta$-chitin from squid pens of the species Dosidicus gigas was performed using a method based on the work of Chaussard and Domard [14]. In brief, the isolation of chitin (squid chitin (CTS)) consisted of washing, grinding and deproteinization. Firstly, squid pens were washed with tap water and ground into particles $1 \mathrm{~mm}$ in diameter. Then, the particles were submitted to deproteinization by treatment with $1 \mathrm{M}$ sodium hydroxide $(\mathrm{NaOH})$ (Panreac Química SAU, Spain) aqueous solution, at a ratio of $1: 15(\mathrm{w} / \mathrm{v})$, for $24 \mathrm{~h}(\mathrm{hrs})$, under stirring, at room temperature (RT). The obtained material was filtered under vacuum and washed several times with distilled water until neutrality was reached, then dried at RT.

2.2.2. Conversion of chitin into chitosan. A deacetylation process was used to convert CTS into CHTS based on the work of Shimojoh et al [10], consisting of the hydrolysis of the acetamide groups of CT by alkaline treatment with a $50 \%$ $\mathrm{NaOH}$ solution, at a ratio of $1: 10(\mathrm{w} / \mathrm{v})$, under reflux between 85 to $100{ }^{\circ} \mathrm{C}$, over $2 \mathrm{~h}$. This alkaline treatment was repeated three times, where the $\mathrm{NaOH}$ solution was replaced each time. Afterwards, the material was filtered and washed until neutral. Finally, the CHTS was frozen at $-80{ }^{\circ} \mathrm{C}$ and freeze-dried (Telstar equipment, Spain) for four days [9].

2.2.3. Purification of chitosan. A purification process was necessary to obtain pure CHT which was used for the production of CHT-based materials such as membranes and scaffolds [15]. Both CHTS and CHTC were purified by a reprecipitation method [15]. CHTS and CHTC were dissolved at concentration of $1 \%(\mathrm{w} / \mathrm{v})$ in $2 \%(\mathrm{v} / \mathrm{v})$ acetic acid (from Pronolab, Portugal) aqueous solution. The system was maintained and stirred overnight at RT. Afterwards, the solution was filtered twice to remove impurities and CHT was then precipitated using $3 \mathrm{M} \mathrm{NaOH}$ solution. The precipitate was separated by filtration, washed with distilled water until neutral and freeze-dried for four days.

\subsection{Characterization of chitin/chitosan}

2.3.1. Determination of molecular weight. The molecular weight of CHTS and CHTC was determined by size exclusion chromatography-multiangle laser-light scattering (SEC-MALLS) [16]. The system was composed of an Iso Pump G1310A Hewlett Packard, with light scattering PSS SLD7000 MALLS detector (Brookhaven Instruments Corporation, USA) operating at $660 \mathrm{~nm}$. Both CHTS and CHTC were dissolved in acetic acid solutions of 0.3 to $2 \mathrm{~g} \mathrm{~L}^{-1}$ and shaken at RT. CHT solutions were filtered with a syringe filter membrane of $0.45 \mu \mathrm{m}$. Analyses were performed at RT in $0.2 \mathrm{M}$ acetic acid buffer solutions and $0.15 \mathrm{M}$ ammonium acetate solution as a mobile phase. Values of $\mathrm{dn} / \mathrm{dC}$ were taken from the literature $[9,16]$.

2.3.2. Determination of particle size. The particle size of CT and CHT was determined by dynamic light scattering (DLS) on a suspension of those materials in water, using Zetasizer ${ }^{\circledR}$ equipment (Malvern, UK).

2.3.3. Measurement of the degree of $N$-deacetylation. The average degree of deacetylation (DD) of the CT and CHT samples was determined from data of elemental analysis and nuclear magnetic resonance spectroscopy (NMR), respectively. Elemental analysis was performed in the Laboratory Analisis Instrumental, (CACTI), University of Vigo, using a Fisons EA-1108 elemental analyser. NMR spectra were recorded using Bruker Avance II $400 \mathrm{~Hz}$. For this purpose, the samples were dissolved in $2 \%$ deuterated acetic 
acid in deuterated water $\left(\mathrm{D}_{2} \mathrm{O}\right)$ solution. After dissolution approximately $1 \mathrm{~mL}$ of the CHT sample solution was transferred to a $5 \mathrm{~mm}$ NMR tube. The sample tube was inserted in a magnet and allowed to reach thermal equilibrium $10 \mathrm{~min}$ before the experiment was performed.

2.3.4. Chemical composition. The chemical composition was characterized by Fourier transform infrared spectroscopy (FTIR), using a Shimadzu IR Prestige 21 spectrometer (from Shimadzu, Europa), in the spectra region of $4000-400 \mathrm{~cm}^{-1}$ with resolution of $16 \mathrm{~cm}^{-1}$ after acquisition of 32 scans. The samples were powdered, mixed with potassium bromide $(\mathrm{KBr})$ and processed into pellets.

\subsection{Preparation of polymeric systems with squid and commercial chitosan}

Polymeric systems, in particular membranes and porous structures, were produced using squid and CHTC. The CHTC was used as reference.

2.4.1. Production of membranes. CHT membranes were prepared by solvent casting [17]. CHTS and CHTC (powder) were dissolved in $2 \%(\mathrm{v} / \mathrm{v})$ acetic acid solution at a concentration of $1 \%(\mathrm{w} / \mathrm{v})$. Then, CHT solutions were poured into Petri dishes and the solvent allowed to evaporate at RT for about four days. In order to neutralize the acetic acid, the dried membranes were immersed in $0.1 \mathrm{M} \mathrm{NaOH}$ solution for about 15 min [18] and then washed with distilled water to remove all traces of alkali, followed by drying at RT.

\subsubsection{Production of porous structures. Porous CHT} structures were developed using the freeze-drying technique $[5,19]$. CHTC and CHTS powders were dissolved in $2 \%$ $(\mathrm{v} / \mathrm{v})$ acetic acid solution at concentrations of 3 and $4 \%$ $(\mathrm{w} / \mathrm{v})$. CHT solutions were then transferred into a silicone mould, frozen at $-80^{\circ} \mathrm{C}$ overnight and freeze-dried for four days to completely remove the solvent, thus producing the desired porous structures. The neutralization of scaffolds was performed using two different methods. In the first method (M1) the samples were immersed in $1 \mathrm{M} \mathrm{NaOH}$ for one hour and washed with distilled water until neutral [20]. In the second method (M2) [20], the samples were soaked in ethanol/water $(\mathrm{Et} / \mathrm{W})$ solutions for $10 \mathrm{~min}$. After that, the scaffolds were immersed in $1 \mathrm{M} \mathrm{NaOH}$ solution for $30 \mathrm{~min}$, and then washed abundantly with distilled water until neutral [21]. The samples were identified as CHTXY, where $\mathrm{X}$ indicates the polymer concentration and $\mathrm{Y}$ the method of neutralization.

\subsection{Characterization of polymeric systems}

2.5.1. Mechanical properties. The mechanical properties of the CHTS and CHTC membranes were characterized by dynamic mechanical analysis, DMA. The viscoelastic measurements were performed using a TRITEC8000B DMA from Triton Technology [10], equipped with the tensile mode. The experiments were performed in wet and dry states, at $37{ }^{\circ} \mathrm{C}$. The thickness of the tested membranes (in the dry state) was: $30.0 \pm 2.1 \mu \mathrm{m}$ and $32.0 \pm 2.9 \mu \mathrm{m}$ for CHTS and CHTC, respectively. The tests were also performed in wet conditions using phosphate buffer saline solution (PBS) and the registered values for thickness were: $60.3 \pm 1.9 \mu \mathrm{m}$ and $42.7 \pm 2.1 \mu \mathrm{m}$ for CHTS and CHTC, respectively. The distance between the clamps was $10 \mathrm{~mm}$ and the membranes samples were cut to about $5 \mathrm{~mm}$ widths, measured accurately for each sample. Four samples were used in each analysis. All samples were analysed in wet conditions, using PBS, placed in a Teflon reservoir, with prior membranes equilibrated for about 50 min in PBS solution. After equilibration at $37^{\circ} \mathrm{C}$, the DMA spectra were obtained during a frequency scan between 0.1 and $20 \mathrm{~Hz}$. The experiments were performed under constant strain amplitude $(30 \mu \mathrm{m})$. A static pre-load of $1 \mathrm{~N}$ was applied during the tests to keep the sample tight. The same conditions were used for the dry experiments, but the membranes were not immersed in the liquid bath. The DMA results were presented in terms of two main parameters: storage modulus $\left(E^{\prime}\right)$ and loss factor $(\tan \delta)$.

The mechanical behaviour of the developed scaffolds in the dry state was assessed under static compressive solicitation. The unconfined static compressive mechanical properties of the scaffolds were measured using an INSTRON 5543 (Instron Int. Ltd, USA) with a load cell of $1 \mathrm{kN}$ for $60 \%$ of strain, at a loading rate of $2 \mathrm{~mm} \mathrm{~min}^{-1}$. The initial linear modulus on the stress/strain curves $(n=6)$, obtained by the secant method, defines the compressive modulus (or Young's modulus).

2.5.2. Structural stability. Structural stability tests were performed by immersing both squid and CHTC membranes and scaffolds in PBS solution ( $\mathrm{pH}$ 7.4) and PBS with $13.6 \mathrm{mg} \mathrm{L}^{-1}$ lysozyme (Sigma Aldrich, Germany), at $37^{\circ} \mathrm{C}$, for up to 60 days, changing the solution every seven days. At the end of each period of time (time point), the samples were removed and washed thoroughly with distilled water. Finally the membranes were dried at $60^{\circ} \mathrm{C}$ and the weight loss was calculated using equation (1):

$$
\text { Weigh loss }=\left[\left(\frac{W_{i}-W_{f}}{W_{f}}\right) \times 100\right]
$$

where $W_{i}$ is the initial weight and $W_{f}$ is the final weight of the sample.

2.5.3. Surface properties. The surface properties of the CHTS and CHTC membranes were assessed by means of static contact angle $(\theta)$ measurements using the sessile drop method with ultra-pure distilled water (polar) and diiodomethane (nonpolar) (OCA equipment, Germany and SCA-20 software), at RT (ca $20^{\circ} \mathrm{C}$ ). At least five measurements were performed for each solvent. Surface tension $(\sigma)$, as well as its polar $\left(\sigma_{p}\right)$ and dispersive $\left(\sigma_{d}\right)$ components, were determined by the Owens and Wendt method [22], using the software G402.

\subsubsection{Study of morphology: micro-computed tomography} $(\mu-c t)$. The microstructure of the scaffolds was evaluated using a high-resolution $\mu$-ct Skyscan 1072 scanner (Skyscan, Kontich, Belgium) with a resolution pixel size of $6.59 \mu \mathrm{m}$ and integration time of $1.9 \mathrm{~s}$. The $\mathrm{x}$-ray source was set at $32 \mathrm{keV}$ 
and $191 \mu \mathrm{A}$. Approximately 500 projections were acquired over a rotation range of $180^{\circ}$ with a rotation step of $0.45^{\circ}$. Data sets were reconstructed using standardized cone-beam reconstruction software (NRecon v1.4.3, SkyScan). The output format for each sample was 500 serial $1024 \times 1024$ bitmap images. A representative data set of 500 slices was segmented into binary images with a dynamic threshold of 57-255 (grey values). The same representative volume of interest (VOI) was analysed for all the samples. These data sets were used for morphometric analysis (ct Analyser, v1.5.1.5, SkyScan) and to build the 3D models (ANT 3D creator, v2.4, SkyScan).

\subsubsection{Study of morphology: scanning electron microscopy.} The morphology of the scaffolds, before and after the neutralization process, was also observed using a XL 30 ESEM-FEG Philips microscope. Longitudinal sections of samples were obtained after being frozen in liquid nitrogen. Prior to SEM analysis, specimens were coated with gold using a Cressington sputter coater and the analysis was performed at an accelerating voltage of $15 \mathrm{kV}$ and magnifications from $50 \times$ to $100 \times$.

2.5.6. Cytotoxicity tests. In order to assess the possible cytotoxicity of the samples, extracts (leachables) of all membranes and scaffolds were evaluated following MEM extraction tests (72 h), according to ANSI/AAMI/ISO-109935 guidelines [23]. For these tests a mouse fibroblast-like cell line, L929 cells, (L929 cells; ECACC, UK) was used and cell metabolic activity was assessed by using a MTS (3-(4,5-dimethylthiazol-2-yl)-5-(3-carboxymethoxyphenyl)2-(4-sulfophenyl)-2H-tetrazolium) assay. In MEM extraction tests, the materials and the control (latex as positive control) were extracted by incubation in complete medium (the same used for cell culture) for $24 \mathrm{~h}$ at $37^{\circ} \mathrm{C}$ and $60 \mathrm{rpm}$ (in thermostatic bath) [24]. After the defined period of incubation, the resulting solution was removed, thus obtaining the extraction fluid. L929 cells were cultured in basic medium: Dulbecco's Modified Eagle's Medium (DMEM, Sigma-Aldrich, USA) with phenol red and supplemented with $10 \%$ foetal bovine serum, FBS (Gibco, UK) and $1 \%$ antibiotic/antimycotic (A/B, Gibco, UK) solution and incubated at $37^{\circ} \mathrm{C}$ in an atmosphere containing $5 \% \mathrm{CO}_{2}$ until achieving $90 \%$ confluence. Then, a cell suspension was prepared with a concentration of $1 \times 10^{5}$ cells $\mathrm{mL}^{-1}$ and seeded on extracts in 24-well plates and incubated for 24, 48 and $72 \mathrm{~h}$. The L929 cells' relative viability (\%) was determined for each extract and compared to tissue culture polystyrene (TCPS), used as a negative control of cell death. Latex extracts were considered as a positive control of cellular death. After each time point extracts of the solutions were removed and the plates were evaluated microscopically (in an inverted phase contrast optical microscope) to assess the confluence of the monolayer and changes in cellular morphology. At each point an MTS test was performed to assess L929 cellular viability. For this assay, an MTS solution was prepared using a 1:5 ratio of MTS reagent and culture medium containing DMEM without phenol red, 10\% FBS and 1\% A/B solution, followed by a $3 \mathrm{~h}$ incubation period at $37^{\circ} \mathrm{C}$. Finally, the optical density (OD) at $490 \mathrm{~nm}$, directly proportional to the concentration of a brown formazan product resulting from the bioreduction of MTS by living cells in culture, was read on a multiwell microplate reader (Synergy HT, Bio-Tek Instruments). All cytotoxicity-screening tests were performed using nine replicates $(n=9)$. The obtained results were compared with controls, namely culture polystyrene (TCPS) as a negative control and latex as a positive control of cellular death.

\subsection{Statistical analysis}

Statistic analysis of the data was performed by hypothesis testing $t$-test using OriginPro 8.0, OriginLab Corporation. Differences between the groups with $p<0.05$ were considered statistically significant.

\section{Results and discussion}

\subsection{Chitosan produced from squid pens}

Despite the increasing attention that marine organisms have been receiving, they are efficiently exploited and residues with valuable compounds are being discarded. An example is the endoskeleton of squid from which CT and consecutively CHT can be obtained. CT was isolated from the endoskeletons of Dosidicus gigas (giant squid) by deproteinization with $\mathrm{NaOH}$ solution, with a yield of $38.6 \%$ in respect to raw material. Further, CTS was converted into CHTS by a deacetylation procedure, as described in the experimental section and several materials were characterized by FTIR. The spectra, in figure $1(A)$, obtained with CTC and CTS, showed the characteristic peaks of chitin at $1660 \mathrm{~cm}^{-1}$ (amide I) and $1580 \mathrm{~cm}^{-1}$ (amide II) [11], which indicated that independently of the source, chitin (CTS and CTC) have the same chemical nature. When comparing the spectra obtained with CHTC and CHTS, in figure $1(B)$, their similarity is noted, where the characteristic peaks of CHT were observed at $1660 \mathrm{~cm}^{-1}$ (amide I) and $1580 \mathrm{~cm}^{-1}$ (amide II) [12], but with different relative intensities than the ones observed in chitin spectra, as highlighted in figure $1(C)$. In particular, the peak observed at $1660 \mathrm{~cm}^{-1}$, attributed to the vibration of $\mathrm{C}-\mathrm{O}$ bonds, is more intense for CT than for CHT because during the deacetylation of chitin $\mathrm{C}-\mathrm{O}$ groups are being removed from the polymer. These findings support the success of the used procedures on the production of chitin and CHT from endoskeletons of Dosidicus gigas.

The performance of CT and CHT in several applications, namely in biomedicine and pharmacy, is influenced by properties such as the size of the polymer chains and the DD [25]. Thus, in order to assess those properties, the average molecular weight of CHTC and CHTS was determined by using SEC-MALLS, which obtained a value of $91.8 \mathrm{kDa}$ for CHTS and $116 \mathrm{kDa}$ for CHTC. Both CTC and CTS were not analysed by liquid chromatography due to their difficult solubilities.

The particle sizes of all the developed materials were determined by dynamic light scattering and values of $282 \mathrm{~nm}$ for CTC, $304 \mathrm{~nm}$ for CTS, $145 \mathrm{~nm}$ for CHTC and $149 \mathrm{~nm}$ 


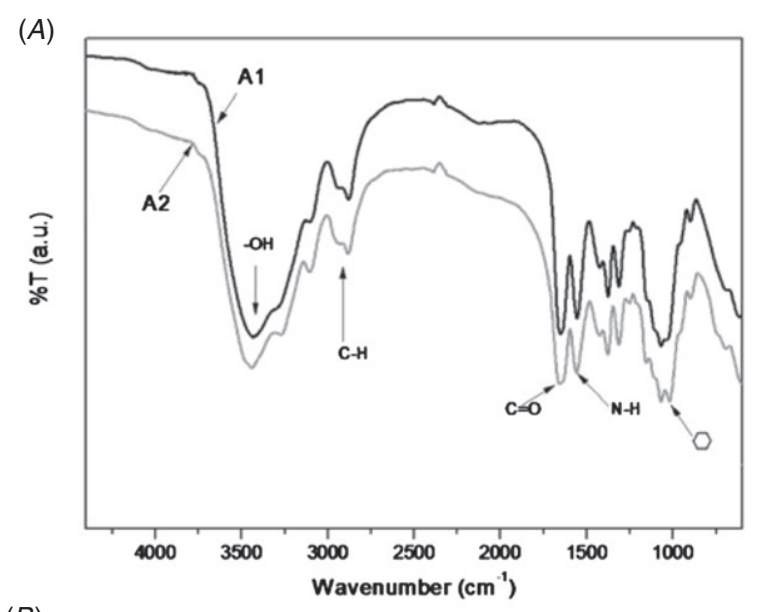

(B)

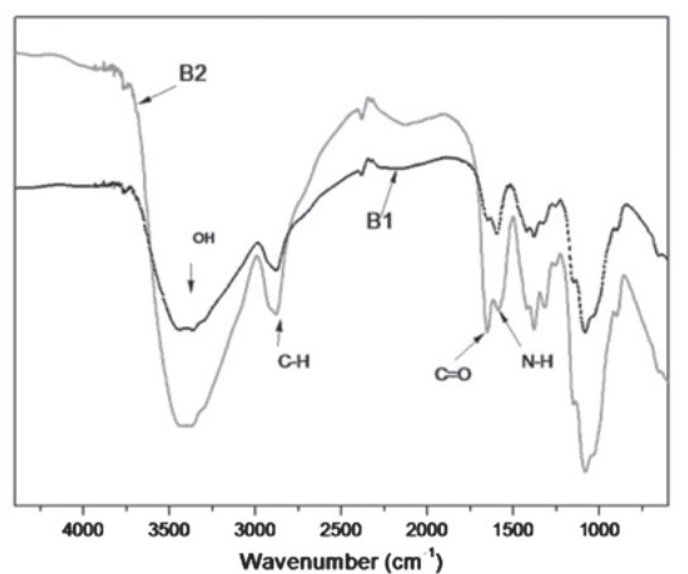

(C)

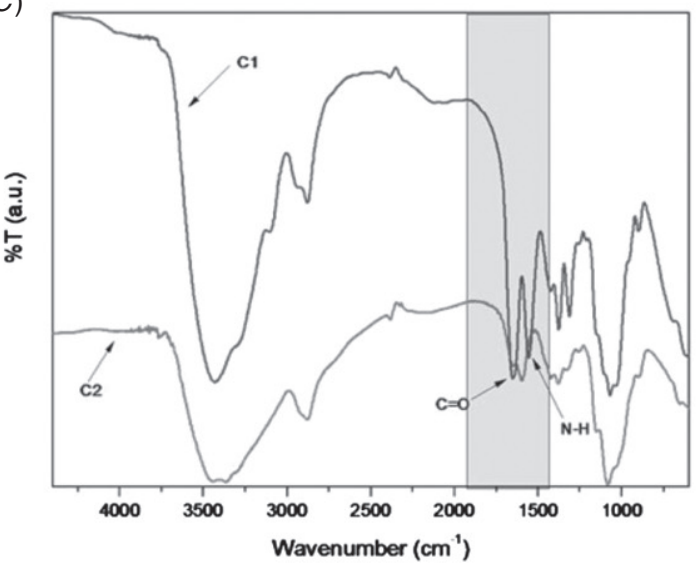

Figure 1. FTIR spectra of $(A)$ chitin (CT), A1-CTS, A2-CTC $(B)$ chitosan (CHT), B1-CHTS, B2-CHTC, $(C)$ C1-CTS and C2-CHTS.

for CHTS were obtained. By these results, CT samples presented a particle size almost the double the one observed for CHT samples. The particle size measured by dynamic light scattering is related to the hydrodynamic volume of the polymer particles, which is dependent on the charge and conformation of the polymer in aqueous suspension [26]. In fact, it can be hypothesized that polymers with higher molecular weights may result in particles with higher hydrodynamic volumes. Thus, one can speculate that $\mathrm{CHT}$ has a significantly smaller molecular weight than CT, which is in agreement with the structural stability of the polymer that may be expected to occur together with the removal of acetyl groups during the chemically aggressive deacetylation process.

The DD, which is the number of glucosamine units of the biopolymer chain with respect to the total number of units [27], is an important property of CHT that dictates some of its physicochemical properties such as solubility, crystallinity, swelling behaviour and material degradation [25]. The obtained DD of the samples were: $42.1 \pm 0.1$ for CTC, $7.2 \pm 0.1$ for CTS, $76.6 \pm 0.4$ for CHTC and $91.2 \pm 0.1$ for CHTS. Both CTC and CTS samples have different values of DD, which can be attributed to the different sources and respective types of CT (crustaceans have $\alpha$-chitin and mollusks have $\beta$-chitin), but also to the isolation processes used in each case. Furthermore, alkaline treatments used for deproteinization can also result in the removal of some acetyl groups and thus increase the DD value. In fact, CTC production follows an industrial-scale process involving a substantial amount of raw material, so control of the process can be difficult. However, the production of CTS following a laboratory-based methodology allows better control of all processing steps [28]. To support this statement, the CHTS has a DD higher than the ones obtained for CHTC. According to the literature [11], both $\alpha$-chitin and $\beta$-chitin show differences in intermolecular forces, where $\beta$-chitin has weaker intermolecular forces. This fact reinforces the susceptibility of $\beta$-chitin to the deacetylation reaction and consequently explains its high DD.

\subsection{Chitosan membranes}

After the successful production of CHT from squid pens, its potential for biomedical applications was assessed through the production and characterization of membranes and porous structures.

CHT membranes were prepared by solvent casting, as described in detail in the Materials and Methods section, and their mechanical properties were studied by DMA. Figure 2 presents the viscoelastic behaviour of membranes prepared with CHTC and CHTS, analysed in dry (A) and wet (B) states. The storage (elastic) modulus $\left(E^{\prime}\right)$ of all studied membranes increased with increasing frequency, with membranes made with CHTS exhibiting the highest $E^{\prime}$ values [4, 29]. This suggests that these membranes possess a higher stiffness than those prepared with CHTC. Figure 2 also presents the variation of the phase angle through $\tan \delta$, with the frequency, that measures the damping properties of the samples and provides an indication of its viscoelastic characteristics [30]. It is observed that both types of membranes present the same behaviour in $\tan \delta$. Since $\tan \delta$ represents the ratio between $E^{\prime \prime}$ and $E^{\prime}$, it can be concluded that the loss modulus was also higher for the membranes prepared with CHTS [31]. Thus, these membranes have also a higher viscous behaviour. Comparison between the results obtained in wet conditions indicated that membranes prepared with CHTS have higher mechanical properties than ones observed on membranes prepared with CHTC. Overall, the values were lower than the ones observed in dry conditions, suggesting that membranes were less stiff in wet conditions probably due 
Table 1. Contact angles $(\theta)$; dispersive $\left(\gamma_{d}\right)$ and polar $\left(\gamma_{p}\right)$ components and superficial energy $(\gamma)$ of the developed membranes (CHTS and CHTS).

\begin{tabular}{lllllll}
\hline Material & & $\theta_{\text {water }}\left({ }^{\circ}\right)$ & $\theta_{\text {diiodomethane }}\left({ }^{\circ}\right)$ & $\gamma_{d}\left(\mathrm{mN} \mathrm{m}^{-1}\right)$ & $\gamma_{p}\left(\mathrm{mN} \mathrm{m}^{-1}\right)$ & $\gamma\left(\mathrm{mN} \mathrm{m}^{-1}\right)$ \\
\hline CHT & CHTC & $99.8 \pm 7.6$ & $61.1 \pm 3.3$ & $26.2 \pm 1.4$ & $2.0 \pm 0.7$ & $28.2 \pm 1.6$ \\
& CHTS & $85.5 \pm 1.1$ & $55.2 \pm 1.7$ & $26.8 \pm 0.9$ & $4.7 \pm 0.7$ & $31.4 \pm 1.1$ \\
\hline
\end{tabular}
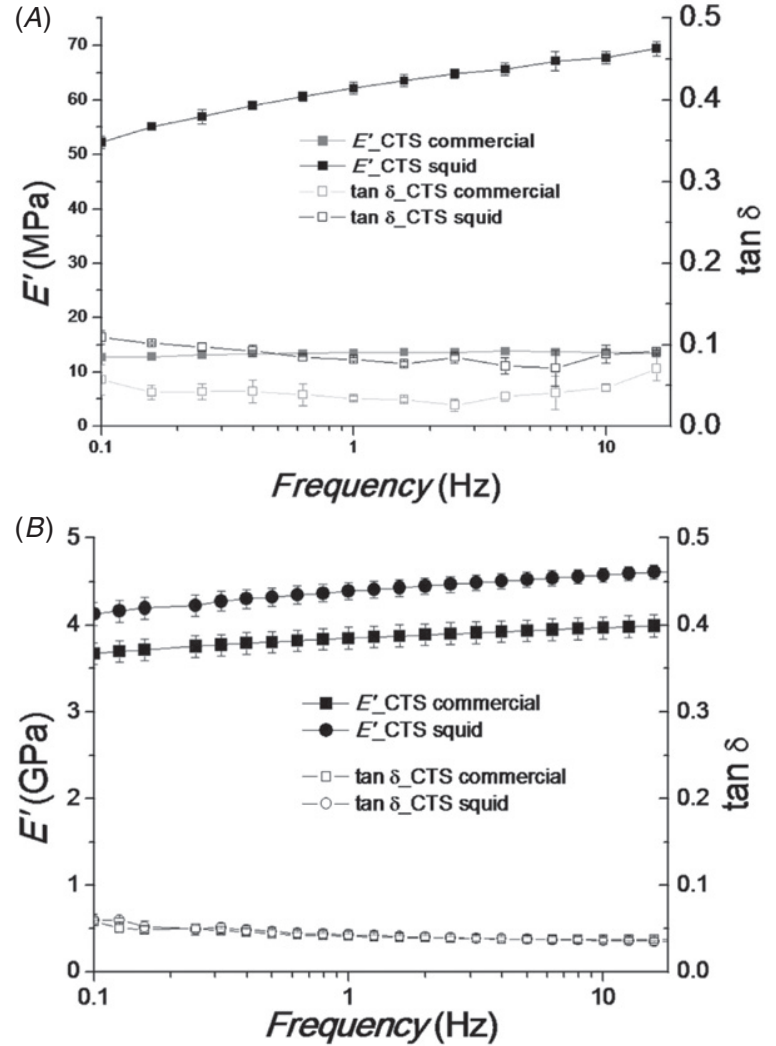

Figure 2. DMA results of CHTS and CHTC membranes: $(A)$ wet state $(B)$ dry state.

to the plasticization effect of water [29, 31]. Regarding viscous behaviour, since $\tan \delta$ was higher for CHTS membranes, the differences in the loss modulus of both membranes should be less pronounced, but the membranes prepared with CHTS were still presenting higher viscous behaviour than the ones prepared with CHTC. These observations are in agreement with others of mechanical strength with DD (between 60 and 100), on CHT swollen with PBS [32].

The surface energy of the prepared CHT membranes was also assessed, in order to characterize their hydrophilicity. The surface energy and contact angles of membranes prepared with CHTS and CHTC were measured by using a contact angle goniometer and the obtained results are summarized in table 1. It can be seen that the CHTS membranes are less hydrophobic than the CHTC membranes. This behaviour is attributed to the higher deacetylation degree of CHTS, since the amino groups have more affinity for water than acetylamine $\left(\mathrm{NHCOCH}_{3}\right)$ and the number of amino groups was higher in CHTS than in CHTC. The surface energy of CHTS membranes was higher than CHTC membranes. However, no significant changes were seen for the non-polar and polar components of surface energy [1].
Table 2. Weight loss of CHTS and CHTC membranes in PBS, in the presence or absence of lysozyme, after 3 to 60 days of incubation at $37^{\circ} \mathrm{C}$.

\begin{tabular}{lllll}
\hline Time (days) & CHTS $^{\mathrm{a}}$ & CHTS $^{\mathrm{b}}$ & CHTC $^{\mathrm{a}}$ & CHTC $^{\mathrm{b}}$ \\
\hline 3 & $7.6 \pm 1.3$ & $9.0 \pm 3.8$ & $18.3 \pm 2.4$ & $15.1 \pm 1.5$ \\
7 & $5.2 \pm 0.7$ & $8.4 \pm 1.4$ & $17.2 \pm 3.7$ & $15.7 \pm 3.5$ \\
14 & $4.8 \pm 1.4$ & $7.8 \pm 2.0$ & $17.0 \pm 1.3$ & $11.5 \pm 2.9$ \\
21 & $5.3 \pm 1.6$ & $8.2 \pm 1.5$ & $11.7 \pm 3.7$ & $12.5 \pm 4.8$ \\
28 & $6.6 \pm 0.2$ & $8.1 \pm 2.5$ & $10.9 \pm 2.5$ & $11.9 \pm 6.4$ \\
45 & $6.6 \pm 0.4$ & $9.2 \pm 0.9$ & $10.6 \pm 0.8$ & $11.7 \pm 1.2$ \\
60 & $2.9 \pm 0.6$ & $4.8 \pm 0.4$ & $11.3 \pm 2.5$ & $12.2 \pm 4.7$ \\
\hline
\end{tabular}

a The samples were immersed in PBS.

b The samples were immersed in lysozyme $\left(13.6 \mathrm{mg} \mathrm{L}^{-1}\right)$.

Membranes for tissue engineering, depending on their composition, are usually expected to degrade after successful tissue regeneration [33]. In this way, it is critical to study the structural stability profile of produced CHT membranes in order to better characterize their potential for application in tissue engineering strategies. The aim of the performed structural stability studies was to simulate physiological conditions using the enzymes present in human serum that would be responsible for enzymatic hydrolysis of CHT materials in vivo, in order to further control and tailor the structural stability of CHT membranes [34]. In vivo, CHT is degraded by enzymatic hydrolysis with lysozyme being the primary agent. This enzyme is present in human serum with a concentration in the range of $7-13 \mathrm{mg} \mathrm{L}^{-1}$ [35]. From this perspective, CHTS and CHTC membranes were incubated in PBS, in the absence (control) and presence of lysozyme, for up to 60 days. The results are shown in table 2. The structural stability profile seems to be stable for at least 60 days of incubation. According to these findings, CHTC membranes are more susceptible to enzymatic structural change, which is most likely due to smaller DD, since the structural stability of CHT appears to be inversely related to the DD [35]. From this perspective, membranes prepared with CHTS are suitable for drug delivery patches and as wound dressings, due to its smaller structural stability rate, which would make these CHT membranes more durable.

\subsection{Porous chitosan structures}

As already mentioned, the potential of CHTS for biomedical applications was also assessed through the production and characterization of porous CHT structures. These porous structures were prepared by freeze-drying CHT solutions with different concentrations, followed by further neutralization with one of two methods, namely $\mathrm{NaOH}$ solution (M1) and ethanol/water prior to treatment with $\mathrm{NaOH}$ solution (M2).

The morphology of porous structures is an important parameter to be considered when an application as scaffold 

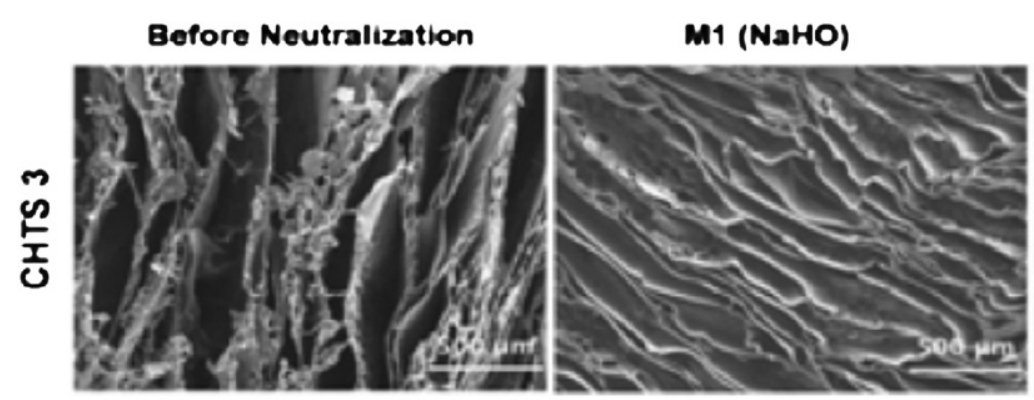

M2 (Ethanol/water)
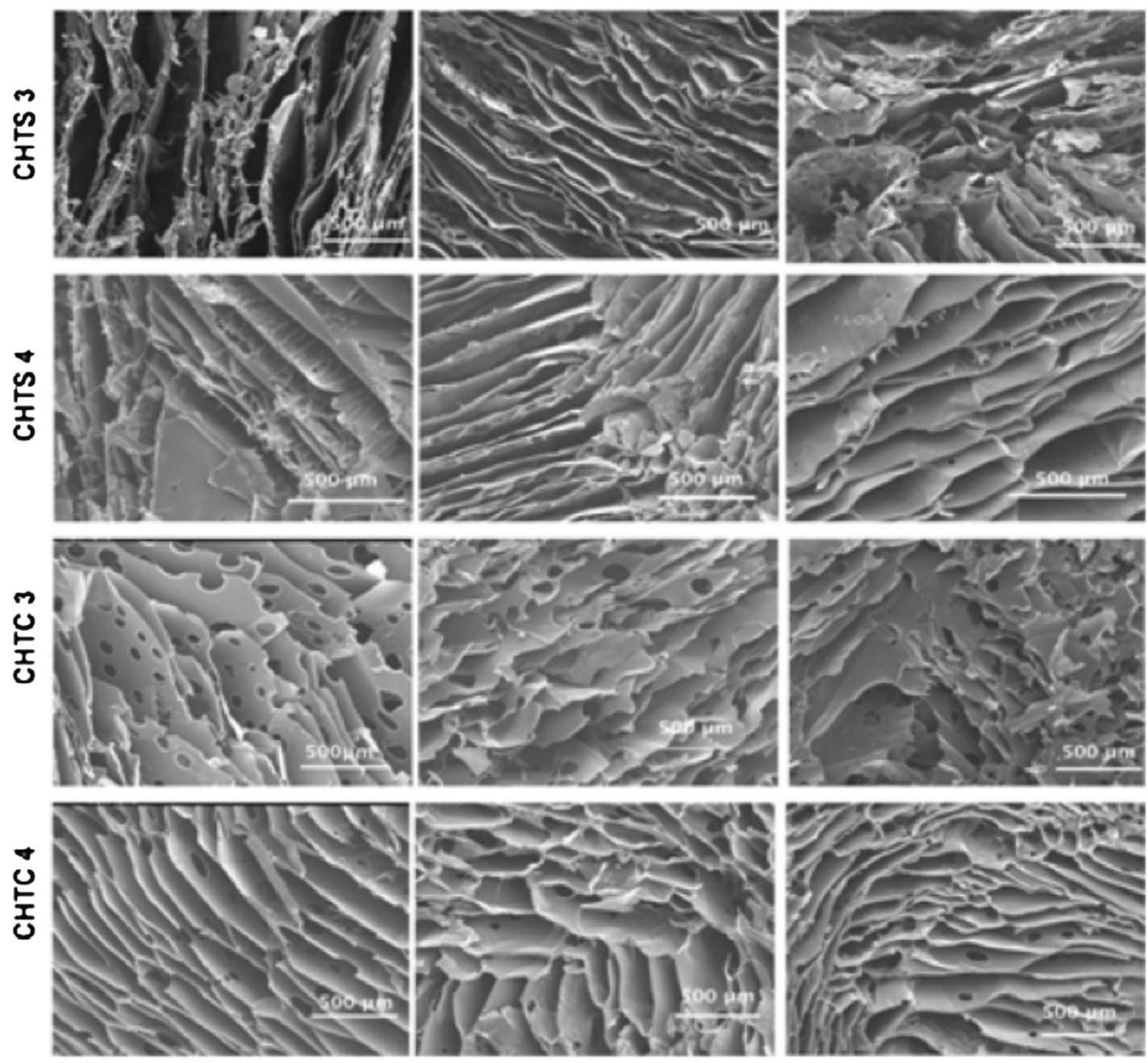

Figure 3. SEM microphotographs of scaffolds prepared with commercial and squid chitosan, using $3 \%$ or $4 \%$ solutions, illustrating their morphology before and after neutralization with method $1-\mathrm{M} 1(\mathrm{NaOH})$ or method 2- $\mathrm{M} 2$ (ethanol/water and $\mathrm{NaOH}$ ).

for tissue engineering is envisaged. Those structures should exhibit a porosity and pore interconnectivity that allows not only the successful seeding of cells, but also their proliferation into the structure [20]. In order to assess the morphology of the prepared CHT scaffolds, SEM analysis has been performed and the obtained images are illustrated in figure 3. Those images show that all scaffolds have welldefined morphology with lamellar features. According to the literature $[20,21]$, CHT based materials need to be neutralized in order to stabilize their structure, and several methods have been proposed, involving the use of ammonia solutions, $\mathrm{NaOH}$ solutions [20], ethanol [20] or mixtures of $\mathrm{NaOH}$ solutions with ethanol [20]. As can be observed in the images depicted in figure 3, before neutralization there are no significant changes in the morphology of CHT scaffolds. Moreover, after neutralization with M1, the lamellar structure was kept constant for all CHT scaffolds. However, when using M2 for the neutralization of scaffolds the lamellar structure was partially destroyed on the scaffolds prepared with CHTC 3 and CHTS 3 (scaffolds prepared with CHTC and CHTS respectively, with concentrations of 3\%) and more significantly when using CHTS. The observed morphological changes are probably due to partial dissolution of the scaffold after contact with ethanol. Regarding the scaffolds prepared from CHT 4 solutions, no significant changes in the lamellar morphology are observed, although when using CHTS 4 the presence of larger pores in the range of 500 to $1000 \mu \mathrm{m}$ was noted. As discussed before, these morphological changes observed in CHTS scaffolds may be due to partial dissolution and a consequential loss of structure, which is in agreement with the expected higher reactivity and interaction of CHTS with solvents reported in the literature [20]. However, the high polymer concentration used in the production of these scaffolds (CHTS 4 and CHTC 4) could also contribute to these results. Overall, freeze-drying CHT solutions conjugated with one of the described neutralization methods can be used to produce porous $\mathrm{CHT}$ structures with lamellar features and pore sizes ranging from $200 \mu \mathrm{m}$ to $1000 \mu \mathrm{m}$, which are adequate for tissue engineering approaches [36]. 
(A)
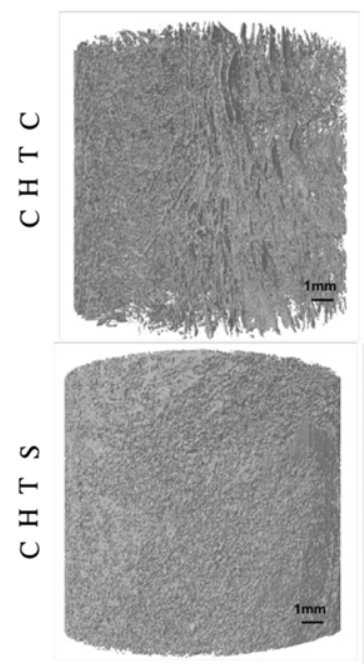

(B)

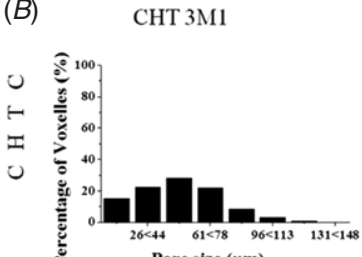

Pore size $(\mu \mathrm{m})$

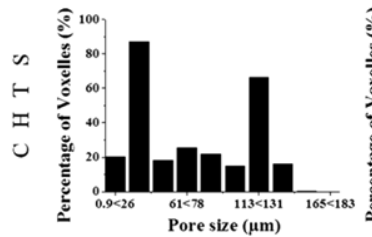

CHT 3M2

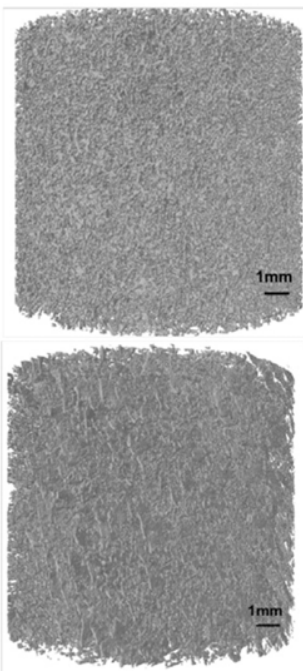

CHT 3M2

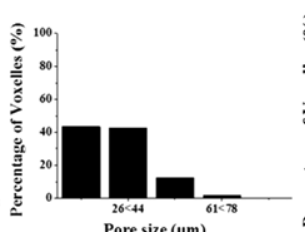

Pore size $(\mu \mathrm{m})$

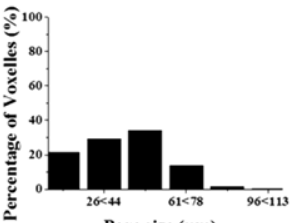

Pore size $(\mu \mathrm{m})$
CHT 4M1
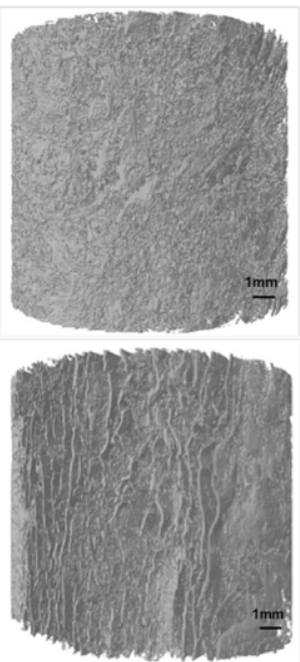

CHT 4MI

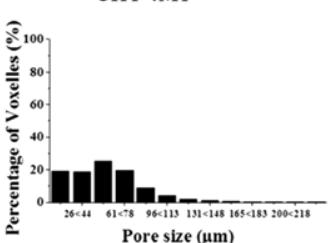

Pore size $(\mu \mathrm{m})$

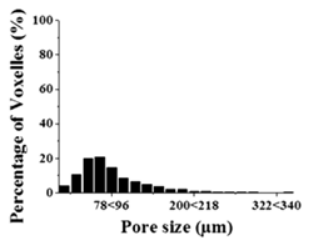

CHT 4M2
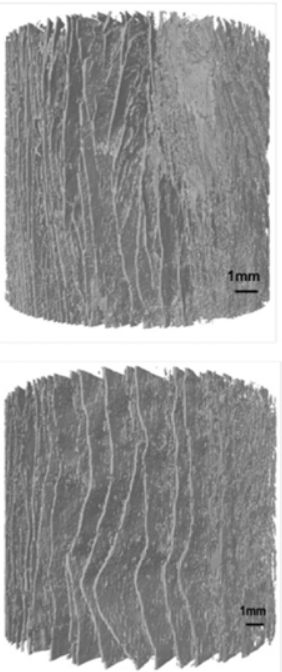

CHT 4M2
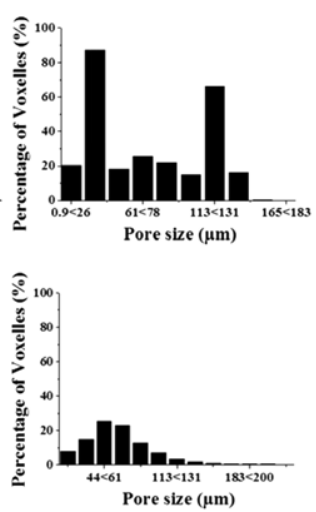

Figure 4. (A) $\mu$-ct 3D microphotographs of scaffolds and $(B)$ mean pore size and distribution of pore size, observed after neutralization.

Table 3. Values of interconnectivity, pore size and porosity for CHTS and CHTC scaffolds before and after neutralization.

\begin{tabular}{llcllrll}
\hline Samples & Properties & CHT 3 $^{\text {a }}$ & CHT3 $^{\text {b }}$ & CHT3 $^{\mathrm{c}}$ & CHT4 $^{\mathrm{a}}$ & CHT4 $^{\mathrm{b}}$ & CHT4 $^{\mathrm{c}}$ \\
\hline CHTC & Interconnectivity $(\%)$ & 88.8 & 81.9 & 87.1 & 3.69 & 68.2 & 36.1 \\
& Pore size $(\mu \mathrm{m})$ & 151.43 & 52.1 & 30.2 & 54.32 & 54.7 & 84.1 \\
& Porosity $(\%)$ & 67.9 & 57.3 & 62.0 & 78.30 & 66.4 & 73.1 \\
CHTS & Interconnectivity $(\%)$ & 2.10 & 73.3 & 93.7 & 1.83 & 50.3 & 60.3 \\
& Pore size $(\mu \mathrm{m})$ & 48.54 & 65.1 & 42.7 & 53.55 & 85.2 & 67.1 \\
& Porosity $(\%)$ & 65.66 & 64.3 & 41.8 & 65.87 & 71.2 & 71.9 \\
\hline
\end{tabular}

${ }^{\text {a }}$ Samples before neutralization.

${ }^{\mathrm{b}}$ Samples neutralized by $\mathrm{M} 1(\mathrm{NaOH})$.

${ }^{\mathrm{c}}$ Samples neutralized by M2 (ethanol/water).

Morphological analysis of the developed CHT scaffolds was also assessed by $\mu$-ct, particularly to characterize pore size, porosity and pore interconnectivity. The 3D $\mu$-ct images, obtained from digital geometry processing from a series of two-dimensional x-ray images, are illustrated in figure 4. Table 3 also presents the determined values of interconnectivity, porosity and pore size for the studied CHT scaffolds. Upon observation of the images of the CHT scaffolds obtained from $\mu$-ct analysis, the porosity seems to be similar for different types and CHT concentrations. From the determined values, it can be stated that scaffolds prepared with both CHTs have higher porosity with increasing concentration of CHT solution, which can be explained by differences in the formation of ice crystals during freezing, due to different concentrations of CHT. It can be concluded that pore size increased with increasing concentration of CHT solution, whereas the interconnectivity decreased. Moreover, these values can be influenced by the neutralization method, which in turn affected the morphological features of the scaffolds. Furthermore, the morphological changes observed for the samples with higher concentrations may be related to the partial dissolution and consequent loss of structure during 


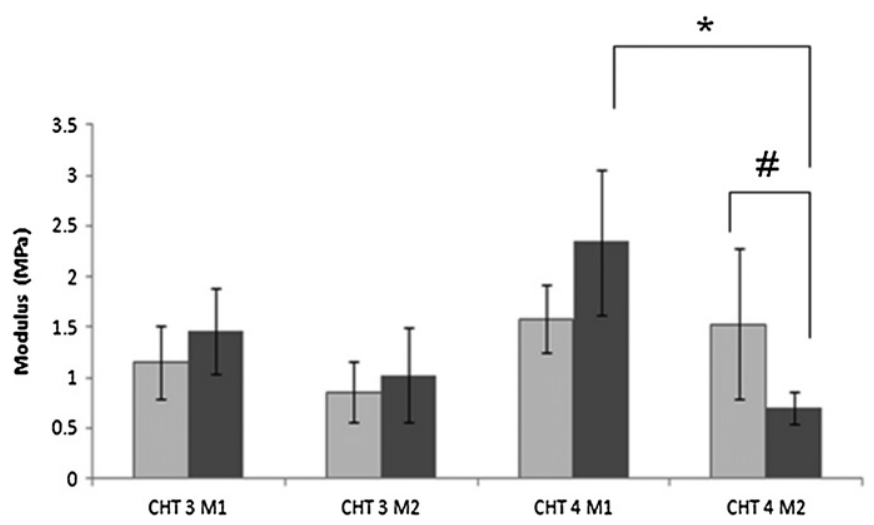

Figure 5. Young's modulus determined from a compression test (dry conditions) to investigate the mechanical properties of porous structures prepared with ( $\square$ CHTC and ( $\square$ CHTS, (*) represents significant differences between scaffold formulations for the same type of chitosan $(p<0.05)$; (\#) represents significant differences between scaffolds prepared with different chitosan origins, but neutralized using the same method $(p<0.05)$.

the neutralization process, namely due to higher reactivity and interactions with solvents such as $\mathrm{NaOH}$ and ethanol, as reported in the literature [19]. Another factor that cannot be neglected is the effect of the cooling rate and thus the solvent crystal growth rate, which influenced the final pore size and porosity of the resulting structure after freeze-drying. Furthermore, the pore size ranged between 30-90 $\mu \mathrm{m}$, which is known to be adequate for tissue engineering purposes [5]. As mentioned before, the neutralization method highly influenced the morphological features of the scaffolds, with CHTC4 M2 scaffolds exhibiting higher porosity and smaller interconnectivity (table 3). Thus, a compromise between the concentration of CHT solutions and neutralization methods must be established in order to render porous structures that exhibit pore sizes that allow cells to populate the interior of the scaffold, but also an adequate interconnectivity that really allows cells to successfully proliferate into the entire polymeric structure [20].

The mechanical properties of those porous structures, in particular the compression modulus, were studied under compression tests in dry conditions. The obtained results for the Young's modulus are illustrated in figure 5.

It was observed that all CHT scaffolds were soft, spongy and very flexible, thus presenting a low resistance to compression. In any case, CHTS scaffolds seem to have better mechanical properties (a higher compressive modulus) than the scaffolds prepared with CHTC, although the observed differences were not statistically significant. These differences may be related to the intrinsic properties of CHT, namely the higher DD of CHTS as observed with CHT membranes, and to the mean pore size of the scaffolds. Moreover, it was observed that the neutralization method also influenced the mechanical properties of the scaffolds. This trend was attributed to the fact that scaffolds neutralized with $\mathrm{NaOH}$ (M1) exhibited some shrinkage, but no significant change in volume or shape. However, scaffolds neutralized firstly with ethanol (M2) exhibited some distortion and partial dissolution, which resulted in structures with higher porosity, thus decreasing their mechanical properties [20]. Of all the prepared scaffolds, the ones presenting better mechanical properties were the ones prepared with a higher concentration of CHTS. The enhanced properties may be due to the reduced formation of ice crystals during freezing as a consequence of the higher concentration of CHT.

As previously stated for CHT membranes, porous CHT structures to be used as scaffolds in TE approaches are expected to degrade during cell proliferation, being substituted by expressed extracellular matrix, thus successfully attaining tissue regeneration [17]. Thus, it is important to characterize the structural stability of CHT scaffolds to understand their potential for application in tissue engineering strategies, particularly because their use will depend on many factors such as the DD, the crystallinity of CHT and the intra- and intermolecular forces present in the polymer. The structural stability of the developed CHT scaffolds was investigated by weight loss after incubation in PBS in the presence of lysozyme, during different time periods, in order to simulate physiological conditions. Incubation in PBS in the absence of lysozyme was also performed as a control. After 60 days of incubation, no significant differences were observed on the weight loss profile of scaffolds prepared with CHTC or CHTS, nor of the scaffolds prepared from CHT solutions of $3 \%$ or $4 \%$. Moreover, no significant differences were observed on the weight loss of scaffolds neutralized by the two proposed methods. To further explore the structural stability and understand its mechanism, additional tests would be needed, including the measurement of molecular weight after incubation and of enzymatic degradation. Based on the weight loss, one can conclude that the structural stability of CHT scaffolds is not significantly affected by the concentration of the solution used to prepare it, nor by the origin of the CHT used, nor even by the neutralization method, within the conditions used in this work.

Besides the physicochemical properties, when considering a material for application in tissue engineering, it is necessary to assess its cytocompatibility under the processing conditions used. From this perspective, the eventual cytotoxicity of the developed CHT scaffolds was evaluated through MTS assay on extracts from those scaffolds, after being in contact with a mouse fibroblast-like cell line, L929 cells, during 24, 48 and 72 h, following established protocols [24]. The obtained results, shown in figure 6 , demonstrate that the developed scaffolds were not cytotoxic and presented cell activity equivalent to that observed with negative controls. Moreover, it is important to highlight that these results show that non-cytotoxic behaviour is independent of the type of CHT used, of the concentration of CHT solution, as well as of the method of neutralization employed. These observations are in accordance to the findings obtained by Chatelet et al [25], where CHT was cytocompatibly independent of the DD.

\section{Conclusions}

Chitin has been successfully isolated from the endoskeletons of the giant squid Dosidicus gigas and further converted into CHT, with a high DD (91\%). Moreover, highly deacetylated 


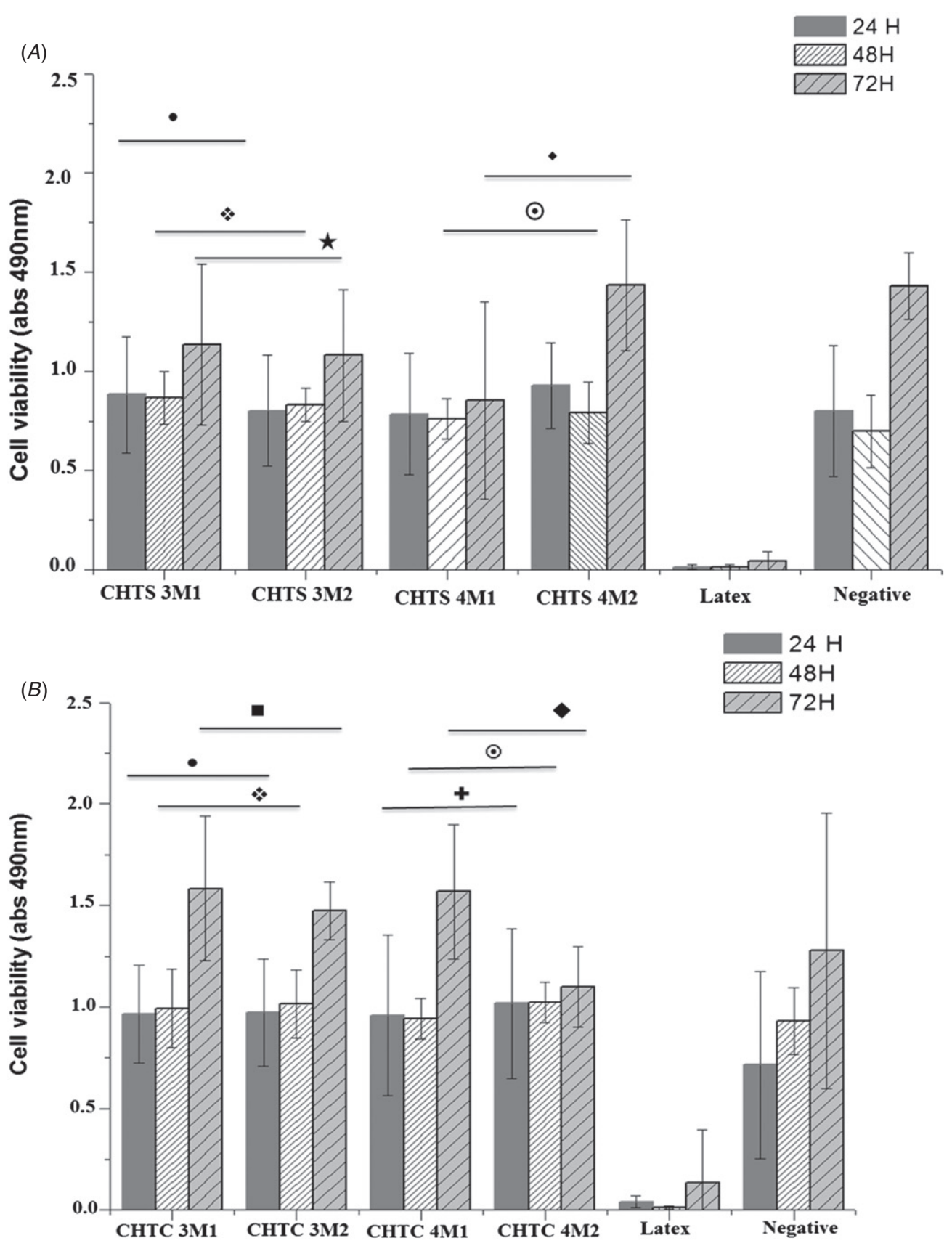

Figure 6. Cytotoxicity assessment with MTS tests on extracts from scaffolds prepared with $(A)$ squid chitosan (CHTS) and (B) commercial chitosan (CHTC). $(\because, \square, \square, \odot$,$) represent significant differences between scaffolds for the same types of chitosan for different types of$ neutralization and the same time of cytotoxicity test $(p<0.05)$.

CHT seems to be easily produced from squid endoskeletons than from crustacean exoskeletons.

With the aim to assess its potential for application in tissue engineering, the produced CHTS was processed into membranes and scaffolds which exhibited equivalent or better properties than the equivalent systems prepared with CHTC. In particular, CHTS systems have similar structural stability and morphological features but better mechanical properties. Regarding cytocompatibility, all produced structures revealed to be non-cytotoxic under the studied conditions. In addition, a new method for the neutralization of CHT structures was proposed, which also leads to higher pore sizes. Thus, a new marine source for the production of a biomedically relevant polymer is presented, reinforcing the potential of marine resources for the biomedical field. Moreover, as the endoskeletons of the giant squid used in this work are obtained from the discards of commercial squid processing, this work has the additional environmental and economical advantage of representing a strategy for the valorization of marine origin residues.

\section{Acknowledgments}

This work was partially funded by FEDER through INTERREG III A Project Proteus and POCTEP Project IBEROMARE. The Portuguese Foundation for Science and 
Technology is gratefully acknowledged for post-doctoral grants of THS, JMO and SSS. The authors would also like to acknowledge to Dr Julio Maroto from the Fundación CETMAR and Roi Vilela from PESCANOVA S.A, Spain, for the kind offer of squid pens and to Dr Ramón Novoa, Professor Ricardo Riguera and Professor Mariana Landín from the University of Santiago of Compostela for the SEC-MALLS measurements.

\section{References}

[1] Mano J F et al 2007 Natural origin biodegradable systems in tissue engineering and regenerative medicine: present status and some moving trends J. R. Soc. Interface 4 999-1030

[2] Silva T H, Alves A, Ferreira B M, Oliveira J M, Reys L L, Ferreira R J F, Sousa R A, Silva S S, Mano J F and Reis R L 2012 Materials of marine origin: a review on polymers and ceramics of biomedical interest Int. Mater. Rev. 57 276-306

[3] Kumar M N V R 2000 A review of chitin and chitosan applications React. Funct. Polym. 46 1-27

[4] Alves N M and Mano J F 2008 Chitosan derivates obtained by chemical modifications for biomedical and environmental applications Int. J. Biol. Macromol. 43 401-14

[5] Oliveira J M, Rodrigues M T, Silva S S, Malafaya P B, Gomes M E, Viegas C A A, Azevedo J T, Dias I R, Mano J F and Reis R L 2006 Novel hydroxyapatite/chitosan bilayered scaffold for osteochondral tissue-engineering applications: scaffold design and its performance when seeded with goat bone marrow stromal cells Biomaterials 27 6123-37

[6] Prabaharan M and Mano J F 2005 Chitosan-based particles as controlled drug delivery systems Drug Deliv. 12 41-57

[7] Li Q, Dunn E T, Grandmaison E W and Goosen M F A 1992 Applications and properties of chitosan J. Biact. Compat. Pol. 7 370-97

[8] Luz G M and Mano J F 2012 Chitosan/bioactive glass nanoparticles composites for biomedical applications Biomed. Mater. 7054104

[9] Tolaimate A, Desbrieres J, Rhazi M and Alagui A 2003 Contribution to the preparation of chitins and chitosans with controlled physico-chemical properties Polymer 44 7939-52

[10] Shimojoh M, Fukushima K and Kurita K 1998 Low-molecular-weight chitosans derived from beta-chitin: preparation, molecular characteristics and aggregation activity Carbohydr. Polym. 35 223-31

[11] Rinaudo M 2006 Chitin and chitosan: properties and applications Prog. Polym. Sci. 31 603-32

[12] Methacanon P, Prasitsilp M, Pothsree T and Pattaraarchachai J 2003 Hetereogeneous N-deacetylation of squid chitin in alkaline solution Carbohydr. Polym. 52 119-23

[13] Rhazi M, Desbrières J, Tolaimate A, Alagui A and Vottero P 2000 Investigation of different natural sources of chitin: influence of the source and deacetylation process on the physicochemical characteristics of chitosan Polym. Int. 49 337-44

[14] Chaussard G and Domard A 2004 New aspects of the extraction of chitin from squid pens Biomacromolecules 5 559-64

[15] Signini R and Campana Filho S P 2001 Características e propiedades de quitosanas purificadas nas formas neutra, acetato e cloridrato Polímeros: Ciência e Tecnologia $1158-64$

[16] Nguyen S, Jolicoeur S H M, Winnik F M and Buschmann M D 2009 Fractionation and characterization of chitosan by analytical SEC and $1 \mathrm{H}$ NMR after semi-preparative SEC Carbohydr. Polym. 75 636-45
[17] Silva S S, Motta A, Rodrigues M T, Pinheiro A F M, Gomes M E, Mano J F, Reis R L and Migliaresi C 2008 Novel genipin cross-linked chitosan-silk fibroin sponges for cartilage engineering strategies Biomacromolecules 2764-74

[18] Hsieh C-Y, Tsai S-P, Ho M-H, Wang D-M, Liu C-E, Hsieh C-H, Tseng H-C and Hsieh H-J 2007 Analysis of freeze-gelation and cross-liking processes for preparing porous chitosan scaffolds Carbohydr. Polym. 67 124-32

[19] Brás A R, Viciosa M T, Dionísio M and Mano J F 2007 Water effect in the thermal and molecular dynamics behavior of poly(L-lactic acid) J. Therm. Anal. Calorim. 88 425-9

[20] Madihally V M and Mathee H W T 1999 Porous chitosan scaffolds for tissue engineering Biomaterials 20 1133-42

[21] Jiankanga $H$, Dichen L, Yaxionga L, Boa Y, Binghenga L and Qina L 2007 Fabrication and characterization of chitosan/gelatin porous scaffolds with predefined internal microstructures Polymer 48 4578-88

[22] Owens D K and Wendt R C 1969 Estimation of the surface free energy of polymers J. Appl. Polym. Sci. 13 1741-7

[23] ISO 2009 Biological evaluation of medical devices:part 5. Test for in vitro cytotoxicity ISO 10993-5

[24] Gomes M E R, Cunha R L, Blitterswijk A M and Bruijn C A 2001 Cytocompatibility and response of osteoblastic-like cells to starch based materials: effects of several additives and processing conditions Biomaterials 22 1911-7

[25] Chatelet C, Damour O and Domard A 2001 Influence of the degree of acetylation on some biological properties of chitosan films Biomaterials 22 261-8

[26] Bender M 1952 The use of light scattering for determining particle size and molecular weight and shape J. Chem. Educ. 29 15-23

[27] Khor E 2001 Chitin: Fulfilling a Biomaterials Promise (Amsterdam: Elsevier) pp 65-72

[28] Sagheer F A A, Sughayer M A A L, Muslim S and Elsabee M Z 2009 Extraction and characterization of chitin and chitosan from marine sources in Arabian Gulf Carbohydr. Polym. 77 410-9

[29] Mano J F, Reis R L and Cunha A M 2002 Dynamic mechanical analysis in polymers for medical applications Polymer Based Systems on Tissue Engineering Replacement and Regeneration vol 86, ed R L Reis and D Cohn (Dordrecht: Kluwer Academic) pp 139-64

[30] Mano J F 2008 Viscoelastic properties of chitosan with different hydration degrees as studied by dynamic mechanical analysis Macromol. Biosci. 8 67-76

[31] Mano J F, Neves N M and Reis R L 2005 Mechanical characterization of biomaterials Biodegradable Systems in Tissue Engineering and Regenerative Medicine ed R L Reis and J San Román (Boca Raton, FL: CRC Press) pp 152-74

[32] Tomihata K and Ikada Y 1997 In vitro and in vivo degradation of films of chitin and its deacetylated derivatives Biomaterials 18 567-75

[33] Charcosset C 2006 Membrane processes in biotechnology: an overview Biotechnol. Adv. 24 482-92

[34] Hirano S, Tsuchida H and Nagao N 1989 N-acetylation in chitosan and the rate of its enzymic hydrolysis Biomaterials 10 574-6

[35] Martins A M, Pereira R C, Leonor I B, Azevedo H S and Reis R L 2009 Chitosan scaffolds incorporating lysozyme into $\mathrm{CaP}$ coatings produced by biomimetric route: a novel concept for tissue engineering combining a self-regulated degradation system with in situ pore formation Acta Biomater. 5 3328-36

[36] Tuzlakoglu K, Alves C M, Mano J F and Reis R L 2004 Production and characterization of chitosan fibers and 3-D fiber mesh scaffolds for tissue engineering applications Macromol. Biosci. 4 811-9 